\title{
Serous Borderline Broad Ligament Tumor/Atypical Proliferative Serous Broad Ligament Tumor
}

National Cancer Institute

\section{Source}

National Cancer Institute. Serous Borderline Broad Ligament Tumor/Atypical

Proliferative Serous Broad Ligament Tumor. NCI Thesaurus. Code C126469.

A rare non-invasive tumor that arises from the broad ligament. It is characterized by the presence of serous epithelial cell proliferation and cytological atypia. 